\title{
Immune Reconstitution Inflammatory Syndrome Associated with Kaposi Sarcoma
}

National Cancer Institute

\section{Source}

National Cancer Institute. Immune Reconstitution Inflammatory Syndrome Associated

with Kaposi Sarcoma. NCI Thesaurus. Code C125712.

A syndrome that occurs in a proportion of patients with HIV infection and Kaposi sarcoma after initiation of highly active antiretroviral therapy. It is characterized by a deterioration in their clinical status, despite control of virologic and immunologic parameters. 